\title{
The incidence of adjuvant chemotherapy-related early-onset cardiac events in breast cancer patients and its relationship with genetic susceptibility: $A$ prospective cohort study
}

\section{Binliang Liu}

National Cancer Center/Cancer Hospital, Chinese Academy of Medical Sciences and Peking Union Medical College https://orcid.org/0000-0002-3692-2272

\section{Xiuwen Guan}

National Cancer Center/Cancer Hospital, Chinese Academy of Medical Sciences and Peking Union Medical College

\section{Yanfeng Wang}

National Cancer Center/Cancer Hospital, Chinese Academy of Medical Sciences and Peking Union Medical College

\section{Xiaoying Sun}

Cancer Hospital of Huanxing

\section{Zongbi Yi}

National Cancer Center/Cancer Hospital, Chinese Academy of Medical Sciences and Peking Union Medical College

\section{Wenna Wang}

National Cancer Center/Cancer Hospital, Chinese Academy of Medical Sciences and Peking Union Medical College

\section{Lixi Li}

National Cancer Center/Cancer Hospital, Chinese Academy of Medical Sciences and Peking Union Medical College

Jingtong Zhai

National Cancer Center/Cancer Hospital, Chinese Academy of Medical Sciences and Peking Union Medical College

\section{Hong Li}

Beijing Anzhen Hospital, Capital Medical University

Fei Ma ( $\square$ drmafei@126.com )

https://orcid.org/0000-0001-9432-1902 
Keywords: Breast cancer, cardiac events, cardiotoxicity, risk factors, single nucleotide polymorphisms Posted Date: March 27th, 2020

DOI: https://doi.org/10.21203/rs.3.rs-18261/v1

License: (c) (1) This work is licensed under a Creative Commons Attribution 4.0 International License. Read Full License 


\section{Abstract}

Background: Although cardiotoxicity has been widely concerned, the study of early-onset cardiotoxicity is insufficient and it is difficult to explain the occurrence of cardiotoxicity only by clinical factors in patients with low cumulative dose of chemotherapy. The purpose of this study was to investigate adjuvant chemotherapy -related cardiac events in breast cancer patients, and to find out the genetic risk factors related to early-onset cardiotoxicity .

Methods: A total of 388 recruited patients completed routine blood, liver and kidney function, D-dimer, TnT, NT-proBNP (or BNP), ECG and UCG tests before and after adjuvant chemotherapy. Twenty-five single nucleotide polymorphisms were tested. Univariable and multivariable analyses were performed to identify independent risk factors.

Results: A total of 256 adjuvant chemotherapy-related cardiac events were recorded in 180 patients (46.4\%), and these included an abnormal ECG (37.4\%), an abnormal UCG (8.8\%), an elevated BNP (5.7\%), and elevated $\operatorname{TnT}(0.3 \%)$. The ATG13 (rs10838611) GG genotype was a protective factor of early-onset cardiotoxicity (10\% reduction in the LVEF) ([OR] $=0.188 ; 95 \% \mathrm{Cl}$ : 0.067-0.525; $\mathrm{P}=0.001)$.

Conclusion: Adjuvant chemotherapy-related cardiac events are quite common in the real world. Genetic factors were closely related to the susceptibility of early-onset cardiotoxicity.

\section{Background}

Chemotherapy-induced cardiac toxicities are one of the most serious side effects of antitumor therapy and have become increasingly recognized in recent years[1]. According to the time of occurrence of cardiotoxic events, it can be classified as acute, early or late[2, 3]. Currently, clinical research on cardiooncology is mainly about late cardiotoxicity, but the understanding of acute or early-onset cardiotoxicity is insufficient. Adjuvant chemotherapy usually ends within 6 months. The adjuvant chemotherapy-related cardiac events is early-onset cardiac events, therefore, it is a good target group to study such cardiotoxicity.

To screen and detect cardiotoxicities, electrocardiogram (ECG), echocardiography (UCG) and biomarkers [troponin I or T, B-type natriuretic peptide (BNP) or N-terminal prohormone of brain natriuretic peptide (NTproBNP)] have their own advantages and disadvantages[2, 3]. A left ventricular ejection fraction (LVEF) > $10 \%$ that decreases to a value below the lower limit of normality suggests cardiotoxicity, thus, the LVEF has become the most commonly used detection index[3].

Late cardiotoxicity was mainly related to high cumulative dosage, however, genetic factors play an important role in the occurrence of cardiotoxicity in patients with low- to moderate-dose of chemotherapy drugs, especially in patients with relatively low cumulative dose of adjuvant therapy[3-6]. Single nucleotide polymorphisms (SNPs) are the research focus for the genetic susceptibility to cardiotoxicity[7]. Currently, cardiotoxicity-related SNPs are mainly found in drug transport, antioxidation, drug metabolism, 
the $\mathrm{NAD}(\mathrm{P}) \mathrm{H}$ oxidase multienzyme complex, DNA repair, the sarcomere structure and function, the topoisomerase- $2 \beta$ expression, cardiomyocyte apoptosis, cardiac autophagy, and intergenic regions[712].

Currently, there are many disadvantages in studying the genetic susceptibility to cardiotoxicity, and these include being a retrospective study, having few samples, having a disunity of the baseline and using different cumulative doses of drugs or treatment methods[7-12]. Therefore, we conducted a prospective cohort study of this study, focusing on early-onset cardiotoxicity. In order to better explore genetic susceptibility, the patients in this study were those who received adjuvant chemotherapy (including trastuzumab) and had not previously received chemotherapy, targeted therapy and radiotherapy.

\section{Methods}

Study subjects and study design

The prospective observational cohort consisted of breast cancer patients who received adjuvant chemotherapy at the National Cancer Center/National Clinical Research Center for Cancer/Cancer Hospital, Chinese Academy of Medical Sciences and Peking Union Medical College between May 2017 and February 2019.

The inclusion criteria of patients in our study were as follows: (1) adult female patients; (2) voluntary participation in this study and voluntary inclusion of their data in this report; (3) pathologically diagnosed with malignant breast cancer; and (4) prepared for using adjuvant chemotherapy. The exclusion criteria were as follows: (1) incomplete basic information of the patient; (2) failed to complete the whole cycle of chemotherapy in our hospital; (3) incomplete routine blood, liver and kidney function, coagulation function, myocardial enzyme (Troponin T), NT-proBNP (or BNP), ECG and UCG tests before and after adjuvant chemotherapy; and (4) failure to detect multiple SNP sites.

This study was approved by the Institutional Review Boards of Cancer Hospital, Chinese Academy of Medical Sciences (No. NCT03537339), and the protocol has been registered on ClinicalTrials.gov with the number NCC201712029.

Adjuvant Treatment

The adjuvant chemotherapy regimens for the enrolled patients were determined by the doctor, and the regimens were as follows:

(1) The AC regimen [epirubicin (EPI) $90 \mathrm{mg} /$ on day 1 and cyclophosphamide (CTX) $600 \mathrm{mg} / \mathrm{on}$ day 1 and repeated every 21 days for 4-6 cycles];

(2) The AC-T $(\mathrm{H})$ regimen and dose-density AC-T(H) [EPI $90 \mathrm{mg} /$ and CTX $600 \mathrm{mg} /$ on day 1, repeated every 14 (dose-density) or 21 days for 4 cycles, followed by paclitaxel (PTX) $175 \mathrm{mg} /$ on day 1 and repeated every 14 days (and trastuzumab $2 \mathrm{mg} / \mathrm{kg}$ on day 1 and repeated every 7 days) for 4 cycles]; 
(3) The TC regimen [docetaxel (DTX) $75 \mathrm{mg} /$ and CTX $600 \mathrm{mg} /$ on day 1, repeated every 21 days for 4-6 cycles];

(4) The TCb(H) regimen [DTX $75 \mathrm{mg} /$ on day 1 (and trastuzumab $2 \mathrm{mg} /$ on day 1 and repeated every 7 days), carboplatin area under receiver-operating curve (AUC) $=5 \mathrm{mg} / \mathrm{mL}$ on day 2 and repeated every 21 days for 6 cycles];

(5) The AT regimen (EPI $75 \mathrm{mg} /$ or on day 1 and PTX $175 \mathrm{mg} /$ on day 2, which was repeated every 21 days for 6 cycles);

(6) The TH regimen (PTX $80 \mathrm{mg} /$ on day 1 and trastuzumab $2 \mathrm{mg} / \mathrm{kg}$ on day 1, which was repeated every 7 days for 12 cycles);

Trastuzumab was used in HER2-positive breast patients, and the dosage and usage of trastuzumab was $4 \mathrm{mg} / \mathrm{kg}$ for the first dose and then $2 \mathrm{mg} / \mathrm{kg}$, which was repeated every 7 days for 1 year or $8 \mathrm{mg} / \mathrm{kg}$ for the first dose, and then $6 \mathrm{mg} / \mathrm{kg}$, which was repeated every 21 days for 1 year.

The AC, AC- $\mathrm{T}(\mathrm{H})$, and AT regimens were classified as anthracycline-containing chemotherapy; the AC-TH, $\mathrm{TCb}(\mathrm{H})$, and $\mathrm{TH}$ regimens were classified as trastuzumab-containing chemotherapy.

Monitoring the cardiac events and cardiotoxicity

All patients should have at least finish the routine blood, liver and kidney function, coagulation function, D-dimer, troponin T, NT-proBNP (or BNP), standard 12-lead ECG (BEIJING FUKUDA FX-7402) and UCG (GE $\mathrm{LOGIQ}^{\mathrm{TM}} \mathrm{E9}$ ) tests before and after adjuvant chemotherapy. The paper speed of ECG was $25 \mathrm{~mm} / \mathrm{s}$. All ECG and UCG records were evaluated by the same radiology team at the National Cancer Center.

The concentration of TnT was detected by an electrochemiluminescence assay, and a troponin T highsensitivity STAT Elecsys platform (Roche ${ }^{\circledR}$ Diagnostics, Manheim, Germany) was applied. The concentration of NT-proBNP was detected by electrochemiluminescence assay using the proBNP II STAT Elecsys platform (Roche ${ }^{\circledR}$ Diagnostics, Manheim, Germany). The cutoff value was defined as the 99th percentile of a healthy population. TnT or NT-proBNP elevation refers to normal before chemotherapy and higher than normal after chemotherapy. All results are recorded in the hospital electronic system for reference.

According to the examination items, the records of cardiac toxicities was divided into four major points: (1) abnormal ECG: nonspecific ST-T segment anomaly; ST-T segment anomaly; sinus arrhythmia; supraventricular arrhythmia; ventricular arrhythmia; low voltage; poor R wave progression; and others; (2) UCG: $10 \%$ reduction in the LVEF (absolute value); (3) elevated TnT levels; (4) elevated BNP or NT-proBNP levels. Cardiac events refer to the occurrence of any one or more of the above. Early-onset is defined as cardiac toxicity during and after adjuvant chemotherapy period. The diagnosis of cardiotoxicity is based on a $10 \%$ reduction in LVEF before and after adjuvant chemotherapy. 
Candidate SNP selection and genotyping

Thirty SNPs from 25 genes were selected from previously reported research and the National Center for Biotechnology Information (NCBI) SNP database (https://www.ncbi.nlm.nih.gov/snp/). The final list of candidate SNPs are shown in Table 1. 
Table 1

The Detection of SNP Site

\begin{tabular}{|c|c|c|c|}
\hline Function & Gene & SNP site & Success rate of detection \\
\hline \multirow[t]{15}{*}{ Drug transport } & ABCA1 & rs3887137 & 97.68 \\
\hline & & rs2235047 & 99.74 \\
\hline & ABCB4 & rs4148808 & 99.48 \\
\hline & $\mathrm{ABCC} 1$ & rs246221 & 99.48 \\
\hline & & rs3743527 & 99.74 \\
\hline & ABCC5 & rs7627754 & 98.71 \\
\hline & SLC22A1 & rs2282143 & 99.48 \\
\hline & SLC22A2 & rs316019 & 98.45 \\
\hline & SLC22A5 & rs2631370 & 50.26 \\
\hline & & rs2631372 & 97.16 \\
\hline & SLC22A17 & rs4982753 & 99.23 \\
\hline & SLC28A1 & rs2290271 & 100.00 \\
\hline & SLC28A3 & rs7853758 & 99.74 \\
\hline & & rs885004 & 100.00 \\
\hline & CNT1 & rs2305364 & 99.23 \\
\hline \multirow[t]{3}{*}{ Antioxidation } & CAT & rs10836235 & 99.74 \\
\hline & GSTP1 & rs1695 & 98.45 \\
\hline & HAS3 & rs2232228 & 99.23 \\
\hline \multirow[t]{3}{*}{ drug metabolism } & CBR3 & rs1056892 & 100.00 \\
\hline & NOS3 & rs1799983 & 99.48 \\
\hline & POR & rs13240755 & 0.00 \\
\hline \multirow[t]{2}{*}{ NAD $(P) H$ oxidase multienzyme complex } & NCF4 & rs1883112 & 99.48 \\
\hline & CYBA & rs4673 & 99.48 \\
\hline \multirow[t]{2}{*}{ DNA repair } & ERCC2 & rs13181 & 97.42 \\
\hline & PRDM2 & rs7542939 & 100.00 \\
\hline Sarcomere structure & CELF4 & rs1786814 & 100.00 \\
\hline
\end{tabular}




\begin{tabular}{|c|c|c|c|}
\hline Function & Gene & SNP site & Success rate of detection \\
\hline Genes with unknown functions & GPR35 & rs3749172 & 97.68 \\
\hline \multirow[t]{2}{*}{ Receptor tyrosine kinase } & \multirow[t]{2}{*}{ ERBB2 } & rs1136201 & 99.23 \\
\hline & & rs1058808 & 100.00 \\
\hline Cardiac autophagy & ATG13 & rs10838611 & 100.00 \\
\hline \multicolumn{4}{|c|}{$\begin{array}{l}\text { ABC, ATP Binding Cassette; SLC, Solute Carrier; CNT1, Concentrative Nucleotide Transporter 1; } \\
\text { CAT,Catalase; GSTP1, Glutathione S-Transferase Pi 1; HAS3, Hyaluronan Synthase 3; CBR3, Carbonyl } \\
\text { Reductase 3; NOS3, Nitric Oxide Synthase 3; POR, Cytochrome P450 Oxidoreductase; NCF4, } \\
\text { Neutrophil Cytosolic Factor 4; CYBA, Cytochrome B-245 Alpha Chain; ERCC2, Excision Repair Cross- } \\
\text { Complementing; PRDM2, PR/SET Domain 2; CELF4, CUGBP Elav-Like Family Member 4; GPR35, G } \\
\text { Protein-Coupled Receptor 35; ERBB2, Erb-B2 Receptor Tyrosine Kinase 2; ATG13, autophagy related } 13\end{array}$} \\
\hline
\end{tabular}

Genomic DNA was extracted from a 1- to 2-mL blood sample that was collected from each patient upon recruitment using a blood DNA kit (BioTeKe Corporation, Beijing, China). A MassARRAY MALDI-TOF System (Sequenom Inc., San Diego, CA, USA) was used to genotype candidate SNPs according to the protocol. Probes were designed according to Assay Design 3.1 (Sequenom Inc.) and synthesized by the Beijing Genomics Institute (Beijing, China).

Purified primer extension reaction products were dispensed onto a 384-well SpectroCHIP bioarray using a MassARRAY Nanodispenser RS1000 (Sequenom Inc.) and determined using a matrix-assisted laser desorption/ionization time-of flight mass spectrometer. Genotype analysis was performed using MassARRAY Typer software version 4.0 (Sequenom Inc.). Duplicate samples and negative controls (without DNA) were used as quality controls for the genotyping. The concordance for duplicate samples was $100 \%$ for all assays. The group information of each sample was concealed for genotyping analysis. Sites with a more than a $95 \%$ successful detection rate were included in the subsequent analysis.

Statistical Analyses

Continuous variables (such as heart rate, QT interval and LVEF) were expressed as the mean \pm SD, and the comparison before and after chemotherapy was performed by

paired-sample t-tests. The Hardy-Weinberg equilibrium test was performed to validate the genotype distributions of each SNP using the $\chi^{2}$ test or Fisher's exact test. Univariable and multivariable analyses were used to identify the significant independent risk factors between the cardiotoxicity and clinical factors or/and genotype distributions of the SNPs. Variables with a $p<0.25[13]$ in the univariate analysis were included in the multivariate analysis. These results are presented as adjusted odds ratios (ORs) with $95 \%$ confidence intervals (Cls). All statistical tests were two-sided, and $p$ values $<0.05$ were considered statistically significant. All analyses above were performed with SPSS software (Version 23, SPSS Inc., IBM, NY, USA). 


\section{Results}

1. Patient characteristics

From a total of 406 breast cancer patients who were admitted to Cancer Hospital, Chinese Academy of Medical Sciences (Beijing, China) between May 2017 and February 2019, 388 patients were finally enrolled. Two patients refused adjuvant chemotherapy, two patients were lost to follow-up during adjuvant chemotherapy, and one patient experienced cancer recurrence during chemotherapy; in addition, multiple SNP sites were unable to be detected in thirteen patients. A flowchart is shown in Fig. 1. All patients were female. The mean (SD) age was 50.5 (10.1) years. The clinicopathologic characteristics of the patients are listed in Table 2. 
Table 2

Demographics and Clinicopathologic Characteristics of Cases

\begin{tabular}{|c|c|c|c|c|}
\hline Demographic or Characteristic & $\begin{array}{l}\text { Total Cases }(\mathbf{N} \\
=388)\end{array}$ & $\%$ & $\begin{array}{l}\text { Cardiac Toxicity } \\
\text { Cases }(\mathrm{N}=180)\end{array}$ & $\%$ \\
\hline \multicolumn{5}{|l|}{ Age } \\
\hline$<60$ & 302 & 77.8 & 142 & 78.9 \\
\hline$\geq 60$ & 86 & 22.2 & 38 & 21.1 \\
\hline \multicolumn{5}{|l|}{ Side } \\
\hline Left & 205 & 52.8 & 98 & 54.4 \\
\hline Right & 178 & 45.9 & 81 & 45.0 \\
\hline Bilateral & 5 & 1.3 & 1 & 0.6 \\
\hline \multicolumn{5}{|l|}{ Stage } \\
\hline I & 107 & 27.6 & 46 & 25.6 \\
\hline II & 182 & 46.9 & 86 & 47.8 \\
\hline III & 99 & 25.5 & 48 & 26.7 \\
\hline \multicolumn{5}{|l|}{ Pathology } \\
\hline Invasive ductal carcinoma & 380 & 97.9 & 179 & 99.4 \\
\hline Non-Invasive ductal carcinoma & 8 & 2.1 & 1 & 0.6 \\
\hline \multicolumn{5}{|l|}{ Molecular subtypes } \\
\hline Luminal A & 32 & 8.2 & 16 & 8.9 \\
\hline Luminal B (HER-2 negative) & 216 & 55.7 & 98 & 54.4 \\
\hline Luminal B (HER-2 postive) & 33 & 8.5 & 11 & 6.1 \\
\hline Her-2 postive & 21 & 5.4 & 13 & 7.2 \\
\hline Triple Negative & 86 & 22.2 & 42 & 23.3 \\
\hline \multicolumn{5}{|l|}{ Operative method } \\
\hline Non breast conserving surgery & 293 & 75.5 & 137 & 76.1 \\
\hline Breast conserving surgery & 95 & 24.5 & 43 & 23.9 \\
\hline \multicolumn{5}{|l|}{ Comorbidity } \\
\hline Hypertension & 82 & 21.1 & 35 & 19.4 \\
\hline
\end{tabular}




\begin{tabular}{|c|c|c|c|c|}
\hline Demographic or Characteristic & $\begin{array}{l}\text { Total Cases }(\mathbf{N} \\
=388)\end{array}$ & $\%$ & $\begin{array}{l}\text { Cardiac Toxicity } \\
\text { Cases }(\mathrm{N}=180)\end{array}$ & $\%$ \\
\hline Diabetes mellitus & 43 & 11.1 & 20 & 11.1 \\
\hline Coronary heart disease & 13 & 3.4 & 4 & 2.2 \\
\hline Hyperlipemia & 19 & 4.9 & 8 & 4.4 \\
\hline Smoking history & 2 & 0.5 & 1 & 0.6 \\
\hline Drinking history & 2 & 0.5 & 1 & 0.6 \\
\hline \multicolumn{5}{|l|}{ Body Mass Index (BMI) } \\
\hline$\geq 28$ & 90 & 23.2 & 47 & 26.1 \\
\hline$<28$ & 298 & 76.8 & 133 & 73.9 \\
\hline Menstrual conditions & & 0.0 & & \\
\hline Premenopausal & 232 & 59.8 & 114 & 63.3 \\
\hline Postmenopausal & 156 & 40.2 & 66 & 36.7 \\
\hline \multicolumn{5}{|l|}{$\begin{array}{l}\text { Adjuvant chemotherapy (Targeted } \\
\text { therapy) regimen }\end{array}$} \\
\hline $\begin{array}{l}\text { Anthracycline + Taxane + } \\
\text { Trastuzumab }\end{array}$ & 25 & 6.4 & 14 & 7.8 \\
\hline Anthracycline + Taxane & 164 & 42.3 & 83 & 46.1 \\
\hline Taxane + Trastuzumab & 40 & 10.3 & 14 & 7.8 \\
\hline Anthracycline Only & 50 & 12.9 & 25 & 13.9 \\
\hline Taxane Only & 109 & 28.1 & 44 & 24.4 \\
\hline $\begin{array}{l}\text { Anthracycline containing } \\
\text { chemotherapy }\end{array}$ & 239 & 61.6 & 122 & 67.8 \\
\hline $\begin{array}{l}\text { Trastuzumab containing } \\
\text { chemotherapy }\end{array}$ & 65 & 16.8 & 28 & 15.6 \\
\hline
\end{tabular}

2. Early-onset Adjuvant Chemotherapy-related Cardiac Events

A total of 256 adjuvant chemotherapy-related cardiac events were recorded for 180 patients (Tables 1 and 2). Patients with cardiac events accounted for $46.4 \%$ of the total population, and 69 patients (17.8\%) had multiple cardiac events.

2.1 ECG recordings 
The heart rate after chemotherapy was significantly higher than that before chemotherapy (before: 75.04 \pm 10.59 vs after: $78.34 \pm 11.10, p=0.000$ ). The QT interval did not change significantly before and after chemotherapy (before: $368.42 \pm 40.50$ vs after: $366.98 \pm 32.90, p=0.519$ ). The relationship between the heart rate before and after chemotherapy and different chemotherapy regimens is shown in Supplementary Fig. 1.

A total of 145 patients had an abnormal ECG, accounting for $37.4 \%$. Among them, 54 patients (13.9\%) had more than one ECG abnormality. The most common ECG abnormality was sinus arrhythmia $(65$, $16.8 \%)$, followed by nonspecific ST-T segment abnormalities $(49,12.6 \%)$ and low voltage of the ECG (45, 11.6\%). All ECG abnormalities are listed in Table 3.

Table 3

Cardiac Toxicity Events Recorded

\begin{tabular}{|llll|}
\hline Examination & Cardiotoxicity & Count & $\%$ \\
\hline ECG & Nonspecific ST-T segment abnormalities & 49 & 12.6 \\
\hline & ST-T segment anomaly & 4 & 1.0 \\
\hline & Sinus arrhythmia & 65 & 16.8 \\
& Supraventricular arrhythmia & 8 & 2.1 \\
\hline & Ventricular arrhythmia & 10 & 2.6 \\
\hline & Low voltage & 45 & 11.6 \\
\hline & Poor R wave progression & 16 & 4.1 \\
\hline UCG & Others & 2 & 0.5 \\
\hline BNP(NT-proBNP)* & I0\% reduction of LVEF(absolute value) & 34 & 8.8 \\
\hline TnT & Increase & 22 & 5.7 \\
\hline *BNP: B-type natriuretic peptide; NT-ProBNP: N-terminal proB-type natriuretic peptide \\
\hline
\end{tabular}

\subsection{UCG recordings}

Reduction in the LVEF was been found in 34 patients which accounted for $8.8 \%$. The left ventricular ejection fraction decreased after adjuvant chemotherapy, but no significant differences were found (before: $71.31 \pm 4.92$ vs after: $70.89 \pm 4.7, p=0.219$ ).

\subsection{BNP (or NT-proBNP) and TnT}

Twenty-two patients (5.7\%) were confirmed to have elevated BNP (or NT-proBNP) levels, and only 1 patient $(0.3 \%)$ had an elevation of TnT after chemotherapy. 


\section{Detection Of SNPs}

See Table 1 for the detection success rate of each SNP. Both rs2631370 and rs13240755 were not included in the analysis because the detection success rate was less than $95 \%$.

\section{Risk Factors Of Potential Early-onset Cardiotoxicity}

Since the current diagnostic criteria of cardiotoxicity are mainly based on the decrease of LVEF, we regard the decrease of LVEF by $10 \%$ as the study end point of potential early-onset cardiotoxicity. The univariate analysis of the early-onset cardiotoxicity risk is shown in Supplementary Table 1. After the multivariate analysis, all clinical risk factors were excluded and only one genetic factor remained: rs 10838611 . The ATG13 (rs10838611) GG genotype was a protective factor of early-onset cardiotoxicity ([OR] $=0.188 ; 95 \%$ Cl: $0.067-0.525 ; \mathrm{P}=0.001$ ) (Table 4). The histograms of the ATG13 (rs10838611) and early-onset cardiotoxicity are shown in Fig. 2.

Table 4

Multivariate Analysis of Risk Factors for Cardiotoxicity

\begin{tabular}{|lllll|}
\hline Risk fators & & OR & Cl & P \\
\hline rs10838611 & GG & 0.188 & $0.067-0.525$ & 0.001 \\
\cline { 2 - 5 } & CG & 0.408 & $0.161-1.034$ & 0.059 \\
& CC & Ref & Ref & Ref \\
\hline
\end{tabular}

\section{Discussion}

At present, the definition of early-onset cardiotoxicity is not uniform, and there are few studies on chemotherapy-related cardiac events or cardiotoxicity in cancer patients undergoing adjuvant chemotherapy, thus, we cannot compare these results with the results of other centers.

Since LVEF is the most important test index according to the definition of cardiotoxicity, we found that LVEF decreased $>10 \%$ in 34 patients (8.8\%). However, more research is needed to determine whether LVEF is reversible in early-onset cardiotoxicity and whether early-onset reduction in LVEF can be used as a valuable indicator for monitoring and a good marker for early intervention were both unknown.

The only related risk factor we found was the genotype of ATG13 (rs10838611), it supports that genetic factors are closely related to cardiotoxicity in patients with ow- to moderate-dose of chemotherapy[4].

As a core member of the ATG family, the function of Atg13/ATG13 has been underestimated at first[14], except to participate in the initiation of autophagy as part of the Unc-51 Like Autophagy Activating Kinase 1 (ULK1) complex (in vertebrates); recent data have identified additional binding partners of Atg13/ATG13, which include Atg8 and Atg17 proteins or acidic phospholipids[14, 15] that have important 
roles in autophagy. The debated correlation found on autophagy-related SNPs suggests that cardiac autophagy may play a complex role in chemotherapy-induced cardiac injury.

The early warning method of cardiotoxicity is limited, which makes patients lose the opportunity for early intervention, also causes great trouble to clinicians. Waiting for cardiotoxicity to occur before treatment intervention is no longer an acceptable strategy[16]. There is a critical need to develop more robust and sensitive measures to identify high-risk groups for chemotherapy-induced cardiotoxicity. How to detect or forewarn cardiotoxicity before the LVEF decreases has been a widely studied problem. It can be seen from our study that SNPs are very valuable as a predictor of early-onset cardiotoxicity. Currently, the most promising indicator is global longitudinal strain (GLS). GLS is a low-cost, noninvasive and more sensitive tool for early subclinical systolic dysfunction than the LVEF and has been proposed for monitoring asymptomatic cardiotoxicity related to chemotherapy[17]. It has been confirmed that the prediction efficiency has been improved significantly by adding GLS to the model that was originally established using only clinical variables[18].

Nevertheless, several limitations exist in the present study. First, all patients were from a single hospital. However, at the National Cancer Center, patients come from all over China, which may minimize this bias. Second, the definition of early-onset cardiac toxicity is not uniform, which is not easy to compare and analyze with previous research.

\section{Conclusion}

Adjuvant chemotherapy-related cardiac events, which was easy to ignore in the past, is quite common in the real world. Genetic factors, like ATG13 (rs10838611) GG genotype, are closely related to the susceptibility of early-onset cardiotoxicity, so it is very valuable for SNP detection to be included in the prediction model.

\section{Abbreviations}

SNP

Single nucleotide polymorphisms

BNP

B-type natriuretic peptide

NT-proBNP

$\mathrm{N}$-terminal prohormone of brain natriuretic peptide

ECG

Electrocardiogram

UCG

Echocardiography

TnT

Troponin T 
ROC

Receiver operating characteristic

AUC

Area under ROC curve

LVEF

Left ventricular ejection fraction

SF

Shortening fraction

EPI

Epirubicin

CTX

Cyclophosphamide

PTX

Paclitaxel

DTX

Docetaxel

OR

Odds ratios

$\mathrm{Cl}$

Confidence intervals

ATG13

Autophagy related 13

ULK1

Unc-51 Like Autophagy Activating Kinase 1

GLS

Global longitudinal strain

\section{Declarations}

\section{Ethics approval and consent to participate}

This study was approved in writing by the Institutional Review Boards of Cancer Hospital, Chinese Academy of Medical Sciences (No. NCT03537339), and the protocol has been registered on ClinicalTrials.gov with the number NCC201712029. All specimens were collected with written informed consent from all subjects enrolled. This study did not interfere with any decisions made by doctors and did not change or delay any treatments.

\section{Consent for publication}

Not applicable. 


\section{Availability of data and materials}

The datasets used and/or analysed during the current study are available from the corresponding author on reasonable request.

\section{Competing Interests}

The authors have declared no conflicts of interest.

\section{Funding}

There are no financial relationships to report concerning the organization that sponsored this research.

\section{Author contributions}

BLL: Conceptualization, data curation, formal analysis, writing-original draft; XWG: Conceptualization and writing-original draft; YFW: Conceptualization and data curation; XYS: Data curation; ZBY: Assistance data analysis; WNW: Conceptualization; LXL: Conceptualization; JTZ: Conceptualization; HL: Methodology and supervision; FM: Conceptualization, methodology and supervision. All authors read and approved the final manuscript.

\section{Acknowledgements}

Not applicable

\section{References}

1. Barish R, Lynce F, Unger K, Barac A: Management of Cardiovascular Disease in Women With Breast Cancer. CIRCULATION2019, 139(8):1110-1120.

2. Armenian SH, Lacchetti C, Barac A, Carver J, Constine LS, Denduluri N, Dent S, Douglas PS, Durand $\mathrm{JB}$, Ewer $\mathrm{M}$ et al: Prevention and Monitoring of Cardiac Dysfunction in Survivors of Adult Cancers: American Society of Clinical Oncology Clinical Practice Guideline. J CLIN ONCOL 2017, 35(8):893911. 
3. Zamorano JL, Lancellotti P, Rodriguez Muñoz D, Aboyans V, Asteggiano R, Galderisi M, Habib G, Lenihan DJ, Lip GYH, Lyon AR et al: 2016 ESC Position Paper on cancer treatments and cardiovascular toxicity developed under the auspices of the ESC Committee for Practice Guidelines. EUR HEART J 2016, 37(36):2768-2801.

4. Blanco JG, Sun C, Landier W, Chen L, Esparza-Duran D, Leisenring W, Mays A, Friedman DL, Ginsberg JP, Hudson MM et al: Anthracycline-Related Cardiomyopathy After Childhood Cancer: Role of Polymorphisms in Carbonyl Reductase Genes-A Report From the Children's Oncology Group. J CLIN ONCOL 2012, 30(13):1415-1421.

5. Visscher H, Ross CJD, Rassekh SR, Barhdadi A, Dubé M, Al-Saloos H, Sandor GS, Caron HN, van Dalen EC, Kremer LC et al: Pharmacogenomic Prediction of Anthracycline-Induced Cardiotoxicity in Children. J CLIN ONCOL 2012, 30(13):1422-1428.

6. Linschoten M, Teske AJ, Baas AF, Vink A, Dooijes D, Baars HF, Asselbergs FW: Truncating Titin ( TTN) Variants in Chemotherapy-Induced Cardiomyopathy. J CARD FAIL 2017, 23(6):476-479.

7. Linschoten M, Teske AJ, Cramer MJ, van der Wall E, Asselbergs FW: Chemotherapy-Related Cardiac Dysfunction: A Systematic Review of Genetic Variants Modulating Individual Risk. Circ Genom Precis Med 2018, 11(1):e1753.

8. Liu B, An T, Li M, Yi Z, Li C, Sun X, Guan X, Li L, Wang Y, Zhang Y et al: The association between earlyonset cardiac events caused by neoadjuvant or adjuvant chemotherapy in triple-negative breast cancer patients and some novel autophagy-related polymorphisms in their genomic DNA: a realworld study. Cancer Commun (Lond) 2018, 38(1):71.

9. Huang KM, Hu S, Sparreboom A: Drug transporters and anthracycline-induced cardiotoxicity. PHARMACOGENOMICS 2018, 19(11):883-888.

10. Force T, Kolaja KL: Cardiotoxicity of kinase inhibitors: the prediction and translation of preclinical models to clinical outcomes. NAT REV DRUG DISCOV 2011, 10(2):111-126.

11. Schneider BP, Shen F, Gardner L, Radovich M, Li L, Miller KD, Jiang G, Lai D, O'Neill A, Sparano JA et al: Genome-Wide Association Study for Anthracycline-Induced Congestive Heart Failure. CLIN CANCER RES 2017, 23(1):43-51.

12. Henriksen PA: Anthracycline cardiotoxicity: an update on mechanisms, monitoring and prevention. HEART 2018, 104(12):971-977.

13. Bursac Z, Gauss $\mathrm{CH}$, Williams DK, Hosmer DW: Purposeful selection of variables in logistic regression. Source Code for Biology and Medicine 2008, 3(1):17.

14. Alers S, Wesselborg S, Stork B: ATG13. AUTOPHAGY 2014, 10(6):944-956.

15. Popelka H, Klionsky DJ: The molecular mechanism of Atg13 function in autophagy induction: What is hidden behind the data? AUTOPHAGY 2017, 13(3):449-451.

16. Carver JR, Szalda D, Ky B: Asymptomatic Cardiac Toxicity in Long-Term Cancer Survivors: Defining the Population and Recommendations for Surveillance. SEMIN ONCOL 2013, 40(2):229-238.

17. Potter, Elizabeth, Marwick, Thomas, H.: Assessment of Left Ventricular Function by Echocardiography The Case for Routinely Adding Global Longitudinal Strain to Ejection Fraction. 
18. Negishi K, Negishi T, Hare JL, Haluska BA, Plana JC, Marwick TH: Independent and Incremental Value of Deformation Indices for Prediction of Trastuzumab-Induced Cardiotoxicity. J AM SOC ECHOCARDIOG 2013, 26(5):493-498.

\section{Supplemental Figure}

Supplementary Figure 1 The heart rates of different chemotherapy regimens. The heart rate after chemotherapy was higher than that before chemotherapy, regardless of chemotherapy regimen.

\section{Figures}

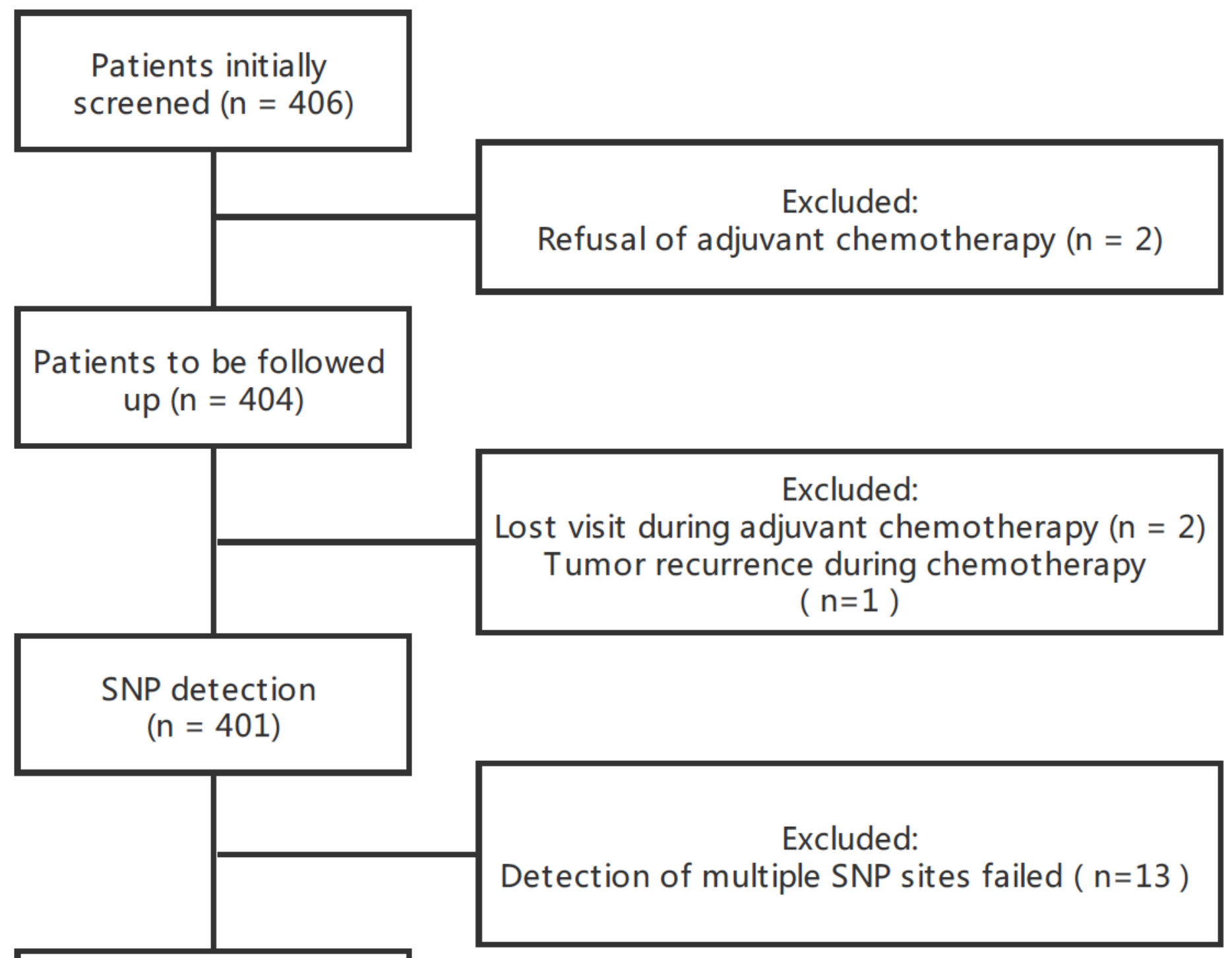

The final inclusion

$$
(n=388)
$$


Figure 1

Flow chart. Flowchart of patient enrollment for our study

\section{rs10838611}

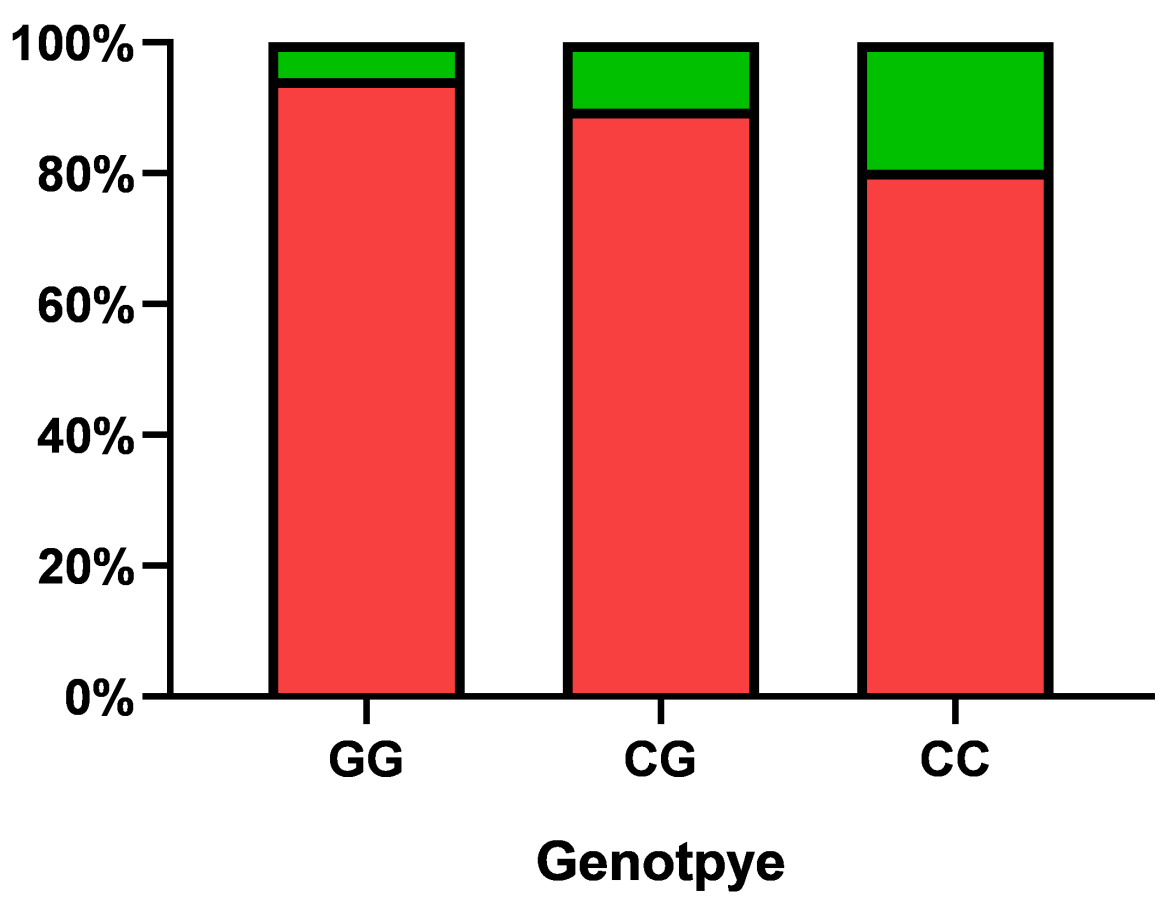

Deduction of LVEF $\square$ Normal LVEF

Figure 2

The proportion of ATG13 (rs10838611) geneotype to early-onset cardiotoxicity. The incidence of earlyonset cardiotoxicity in patients with ATG13 (rs10838611) GG gene was significantly higher than that of the other two genotypes ([OR]=0.188; 95\% Cl: 0.067-0.525; $\mathrm{P}=0.001)$.

\section{Supplementary Files}

This is a list of supplementary files associated with this preprint. Click to download.

- STROBEchecklistcohort.doc

- supFigure1.HeartRatesandchemothreapy.tif 